\title{
Praktische Herausforderungen und Zukunftsvisionen im internationalen Personen- und Güterverkehr - Eine Podiumsdiskussion
}

\author{
Eva Maria Huber
}

Am 1. Februar 2019 fand an der Universität Innsbruck eine moderierte Podiumsdiskussion statt, im Rahmen derer Expertinnen und Experten aus der Transportwirtschaft zum gedanklichen Austausch über den Personen- und Güterverkehr in Europa zusammenkamen. Die Veranstaltung diente nicht nur der Bestandsaufnahme, auch Zukunftsvisionen für den Personen- und Güterverkehr wurden ausgiebig diskutiert. Dazu zeigten die Teilnehmenden auf, in welchen Bereichen aus praktischer Sicht derzeit akuter Regelungsbedarf besteht. Daneben sorgte auch die Mobilität in der Europaregion Tirol-Südtirol-Trentino bei allen Teilnehmenden für Diskussionsstoff. Insgesamt konnten viele wertvolle Erkenntnisse gesammelt werden, die hier dokumentiert und im Anschluss aus rechtswissenschaftlicher Sicht bewertet werden sollen. Zunächst werden die einzelnen Diskussionsbeiträge kurz dargestellt.

Klaus Garstenauer ${ }^{1}$ bezeichnete den Schienenpersonenverkehr in Österreich aufgrund der steigenden Zahl an Bahnreisenden als Wachstumsmotor. Den Zuwachs an Reisenden führte Garstenauer vor allem auf den zunehmenden Standard der von den ÖBB angebotenen Dienstleistungen zurück, durch welchen das Bahnfahren in Österreich attraktiver werde. Garstenauer betonte in diesem Zusammenhang die Bedeutung von Partnerschaften wie jene mit dem Verkehrsverbund Tirol (VVT), zumal erstklassige Dienstleistungen im Eisenbahnsektor seines Erachtens nur in Kooperation angeboten und erbracht werden können. Ein Vorzeigebeispiel sei für ihn der Regionalverkehr in Tirol; dort könne man auf ein dichtes Angebot in einer hohen Qualität blicken, welches eben auch entsprechend Anklang finde. Garstenauer sprach ferner über den grenzüberschreitenden Schienenpersonenverkehr in der Europaregion Tirol-Südtirol-Trentino, dessen Ausbau er aufgrund des Bedarfs

${ }^{1}$ Leiter der Abteilung Nah- und Regionalverkehr, ÖBB-Personenverkehr AG, Wien.

\footnotetext{
E. M. Huber $(\bowtie)$

Institut für Zivilrecht, Universität Innsbruck, Innsbruck, Österreich

E-Mail: eva.m.huber@uibk.ac.at
}

S. Laimer, C. Perathoner (Hrsg.), Mobilitäts- und Transportrecht in Europa,

Bibliothek des Wirtschaftsrechts 2,

https://doi.org/10.1007/978-3-662-63635-0_16 
nach Mobilität grundsätzlich offen gegenübersteht. Gleichzeitig betonte er jedoch nachdrücklich, dass Schritte in diese Richtung angesichts des derzeit mit dem grenzüberschreitenden Schienenpersonenverkehr verbundenen Organisationsaufwandes $^{2}$ nur im Wege von länderübergreifenden Kooperationen denkbar seien. Im Hinblick auf das vielfach diskutierte Thema „Brennerbasistunnel“ (BBT) zeigte Garstenauer verschiedene Probleme auf. So wies er darauf hin, dass der BBT nach österreichischen Normalien errichtet werde, ein Teil des Bauwerks aber auf italienischem Staatsgebiet liege; zur Lösung des Territorialitätskonflikts sei ein Staatsvertrag zwischen Italien und Österreich erforderlich. Zudem stellte er den ersten Bahnhof nach dem BBT (Franzensfeste) ins Zentrum der Diskussion, dem seines Erachtens die für einen Grenzbahnhof typischen Anlagen fehlen. Garstenauer kritisierte zudem das Fehlen einer länderübergreifenden Regelung über die Lenkberechtigung des Lokführerpersonals; nach der derzeitigen Rechtslage müsse ein auf italienischem Staatsgebiet operierender Lokführer sowohl über die einschlägige italienische Ausbildung als auch über italienische Sprachkenntnisse verfügen. Umgelegt auf den BBT bedeute dies, dass eine österreichische Lokomotive (mangels Grenzbahnhofs an der Staatsgrenze) bereits ab Innsbruck zusätzlich mit einem italienischen Lokführer besetzt werden muss, was unökonomisch sei. Harmonisierte Regelungen sind aus seiner Sicht daher vor allem in diesen Bereich dringend notwendig. Im Weiteren äußerte sich Garstenauer zur Verkehrsverlagerung von der Schiene auf die Straße, dem sogenannten „Modal Shift“, wofür die Bahn in den kommenden Jahren die Kapazitäten herstellen müsse. Abschließend richtete Garstenauer seinen Blick in die Zukunft des Mobilitätswesens, welche er ohne die Verwendung von fossilen Energien sieht. Seiner Einschätzung nach werde Mobilität künftig weitgehend ohne eigenes Fahrbetriebsmittel auf hohem Komfort und in einer großen Wahlfreiheit ermöglicht werden.

Astrid Huez $^{3}$ beleuchtete die Herausforderungen für die Transportwirtschaft in Bezug auf den Güterverkehr am Brenner. Ihr zufolge habe der Straßenverkehr in der Region in den vergangenen Jahren aus verschiedenen Gründen stark zugenommen, wodurch die Zahl der Staus steige. Dies belaste aus ihrer Perspektive nicht nur die Umwelt, jeder Stau wirke sich vielmehr auch negativ auf die Konkurrenz- und Wettbewerbsfähigkeit eines Transportbetriebes und so mittelbar auf den gesamten Wirtschaftszweig aus. Problembewusstsein weckte Huez auch in punkto „Fahrerpersonal“, wobei sie vor allem auf den - schon vor Jahren absehbaren - Mangel an Fahrzeugführern und dessen Folgen für die Branche hinwies. Zum einen stiegen dadurch die Personalkosten, weil die Nachfrage an Fachkräften das vorhandene Angebot übersteige; zum anderen könne ein Teil der Lieferungen in Stoßzeiten wie Weihnachten oder Ferragosto mangels ausreichender Kapazitäten nicht (rechtzeitig) erfolgen. Huez führte außerdem vor Augen, dass viele Unternehmen infolge der limitierten Personalressourcen zum Rückgriff auf Fahrerpersonal aus osteuropäischen

\footnotetext{
${ }^{2}$ Wozu etwa die Kenntnis und Implementierung der in anderen Staaten einschlägigen Rechtsvorschriften sowie die entsprechende Schulung des Personals zählen.

${ }^{3}$ Geschäftsführerin der Firma Alpentrans Logistics und Präsidentin des Konsortiums TransOpt Logistic, Bozen/Salurn.
} 
Staaten wie Rumänien oder Bulgarien gezwungen seien, wodurch die Qualität des Personals wegen der schlechteren Grundausbildung zumeist abnehme und Sprachbarrieren zwischen Fahrer und Transportunternehmen entstehen. Auch die Rahmenbedingungen für den Gütertransport am Brenner hätten sich laut Huez in den vergangenen Jahren erheblich verändert, Transportunternehmen müssten heutzutage verschiedenste Verkehrsmaßnahmen - etwa Fahrverbote oder Blockabfertigungen bei der Transportplanung berücksichtigen. Vor diesem Hintergrund spiele man in Fachkreisen mit dem Gedanken, den Transport von der Straße auf die Schiene zu verlagern, um den Kunden ihre Lieferungen trotz eingeschränkter Aktionsradien innerhalb einer bestimmten Zeitspanne zu garantieren. In diesem Zusammenhang bemängelte Huez die fehlenden Güterverladeterminals in Südtirol, zumal damit nach Fertigstellung des BBT ein Verlust an Wettbewerbsfähigkeit für die dort ansässigen Unternehmen einhergehe. Ganz allgemein nimmt die Bahn für Hиеz eine entscheidende Rolle im Transportwesen von morgen ein, ihrer Einschätzung nach sei jedenfalls ein Drittel der Transporte über den Brenner für die Verlagerung auf die Schiene geeignet. Für Huez könnte die Verlagerung auch die Folgen des Fahrzeugführermangels ein Stück weit abfedern. Abschließend forderte Huez zur Auseinandersetzung mit neuen Technologien, z. B autonomes Fahren und Platooning, auf.

Alexander Jug g $^{4}$ stellte zu Beginn das weitreichende Tätigkeitsfeld des Verkehrsverbundes Tirol (VVT) vor, zu welchem die Koordination, die Planung und die Finanzierung des öffentlichen Personenverkehrs, das Vergabewesen, das Marketing, der Vertrieb und die Kundeninformation gehören. Anschließend sprach er über das Anliegen des VVT, die Bevölkerung für den öffentlichen Personenverkehr zu begeistern. Der Umstieg auf die öffentlichen Verkehrsmittel ist laut Jug vor allem zur Realisierung des vom Land Tirol - im Rahmen des Projektes „Tirol 2050“ - angestrebten Ziels der Energieautonomie notwendig, zumal der Verkehr für einen Großteil des Energieverbrauches verantwortlich sei. Jug äußerte sich auch zum öffentlichen Personenregionalverkehr in der Europaregion Tirol-Südtirol-Trentino, für den sich VVT auch weiterhin gemeinsam mit seinen Partnern einsetzen werde, ua sei für 2020 die Einführung eines Stundentaktes nach Südtirol ohne Umstieg am Brenner geplant. Der voranschreitenden Digitalisierung blickt Jug positiv entgegen, ua weil er sich davon einen vereinfachten Zugang zum öffentlichen Personenverkehr - z. B durch Echtzeitfahrplaninformationen - erhofft. Jug beleuchtete auch die Wechselwirkungen zwischen dem Tourismus und der Transportwirtschaft. Ihm zufolge verfügen heutzutage immer weniger Touristen über ein Fahrzeug, was sich auf die Wahl des Urlaubsortes auswirkt. Für diesen Kundenkreis sei nämlich entscheidend, ob sowohl die Anreise als auch die Mobilität am Urlaubsort mit öffentlichen Verkehrsmitteln gewährleistet ist. Aus diesem Grund biete der VVT entsprechende Mobilitätskonzepte für den Tourismus an, wofür dieser im Gegenzug häufig einen erheblichen Teil der mit der Umsetzung verbundenen Kosten trage. Wie Jug betonte, profitiere letztlich jedoch auch die ortsansässige Bevölkerung von

\footnotetext{
${ }^{4}$ Geschäftsführer des Verkehrsverbundes Tirol (VVT), Innsbruck.
} 
solchen Projekten. Die Zukunft des Personennaheverkehrs verbindet Jug mit den Begriffen „Nachhaltigkeit“, „Individualität“" und „einfacher Zugang“.

Marco Kampp ${ }^{5}$ erklärte, dass sich in der europäischen Eisenbahnindustrie wegen der derzeit (noch) fehlenden Standardisierung kein ähnlicher Wettbewerb wie in der Flugindustrie entwickelt, wobei er die Problematik mithilfe von mehreren Beispielen veranschaulichte. So sei allein die Eisenbahninfrastruktur mit rund vier verschiedenen Stromsystemen und rund 20 verschiedenen Signalsystemen europaweit sehr unterschiedlich aufgebaut. Dazu gebe es erhebliche Unterschiede in den nationalen Zulassungssystemen, was etwa zur Folge habe, dass die von den ÖBB verwendete Taurus-Lokomotive aufgrund den mancherorts vorgesehenen Geschwindigkeitsbeschränkungen für Lokomotiven nicht in allen europäischen Staaten zugelassen sei. ${ }^{6}$ Außerdem sei die Zulassung von Lokomotiven in bestimmten Staaten nur streckenbezogen möglich, wodurch das Zulassungsverfahren für jedes einzelne Fahrzeug auf jeder einzelnen Strecke gesondert durchzuführen sei. Dazu zwingen die Anforderungen an das Lokführerpersonal die Bahngesellschaften laut Kampp häufig zu Lokführerwechseln an der jeweiligen Staatsgrenze, wobei er in diesem Zusammenhang beispielhaft auf die Rechtslage in Italien verwies. Da der Umgang mit diesen und anderen nationalen Unterschieden im Eisenbahnwesen sehr kostspielig sei, gebe es, wie Kampp wiederholte, im Schienenverkehr - im Gegensatz zum Flugverkehr, wo einheitliche Standards existieren - bislang eben keinen Wettbewerb. Unter dem Kostenaspekt veranschaulichte Kampp auch die Bedeutung von Kooperationen im internationalen Fernverkehr. Im Alleingang könne den Kunden wegen der kostspieligen Organisation bloß eine bescheidene Zahl an grenzüberschreitenden Schienenverbindungen angeboten werden. Da ein überschaubares Angebot aus Kundensicht jedoch kaum wahrgenommen werde, verringere sich der Umsatz, was letztlich wiederum Einsparungen und die weitere Verkleinerung des Angebotes zur Folge habe. Ein umfangreiches Angebot an grenzüberschreitenden Schienenverbindungen könne deshalb derzeit nur in Zusammenarbeit geschaffen werden. Kampp hob schließlich auch die Schlüsselrolle der Europäischen Union im staatenübergreifenden Schienenverkehr hervor. Seiner Ansicht nach war die Marktliberalisierung ein wichtiger Impuls für die technische Liberalisierung, allerdings hinke letztere der ersten um zehn bis fünfzehn Jahre hinterher. Dies ist für ihn insofern bedenklich, als die Marktliberalisierung ohne die technische Liberalisierung nicht in vollem Maße verwirklicht werden kann. Im Hinblick auf das European Train Control System (ETCS) kritisierte Kampp die beschränkte Kompatibilität der einzelnen Versionslevel. Von der Zukunft erhofft er sich harmonisierte europäische Standards im Eisenbahnwesen, damit eine Zugfahrt - im übertragenen Sinn - von Berlin nach Palermo nahtlos fortgesetzt werden kann.

\footnotetext{
${ }^{5}$ Leiter der Abteilung internationaler Fernverkehr DB Fernverkehr AG, Frankfurt am Main und CEO bei DB Bahn Italia S.r.l., Verona.

${ }^{6}$ Kampp verwies in diesem Zusammenhang auf die Staaten Polen, Tschechien und Italien, wo die Zulassung von Lokomotiven auf eine Geschwindigkeit von $160 \mathrm{~km} / \mathrm{h}$ beschränkt sei. Da die Taurus-Lokomotive im Normalbetrieb eine Geschwindigkeit von $230 \mathrm{~km} / \mathrm{h}$ erreiche und somit über der in diesen Staaten erlaubten Geschwindigkeit liege, sei ihre Zulassung dort nicht möglich.
} 
Insgesamt hat die Veranstaltung wohl eines deutlich gezeigt: Das Transportwesen unterliegt derzeit in mehrfacher Hinsicht einem Wandel. Zum einen zeichnet sich sowohl im Personen- als auch im Güterverkehr ein Trend zur Nutzung von nachhaltigeren Verkehrsmitteln ab, zum anderen wird die Digitalisierung die Branche in den nächsten Jahren stark beschäftigen. Vor allem im zuletzt genannten Bereich sind auch die Rechtswissenschaft und erforderlichenfalls auch der Gesetzgeber gefragt. Eine klare Rechtslage und die damit einhergehende Rechtssicherheit ebnet nämlich erst den Weg für den flächendeckenden Einsatz von neuen technischen Errungenschaften. Dazu ist in diesem Bereich künftig wohl nicht nur die Zusammenarbeit mit anderen Wissenschaftsdisziplinen, sondern auch die enge Vernetzung mit der transportwirtschaftlichen Praxis geboten. Kooperation ist auch weiterhin das Stichwort, wenn man auf das internationale Transportwesen blickt. Vor allem der grenzüberschreitende Schienenverkehr wird aufgrund der derzeit bestehenden nationalen Unterschiede wohl zumindest auf kurz- und mittelfristige Sicht weiterhin nur in Form von Kooperationen der Bahngesellschaften durchführbar sein. Langfristig gesehen wären in diesem und in anderen Bereichen des internationalen Transportrechtes aber jedenfalls - weitere - einheitliche Regelungen wünschenswert. Ein beliebtes Instrument zur Vereinheitlichung des Transportrechtes wird auf globaler Ebene wie schon bislang wohl auch künftig der Staatsvertrag bleiben. ${ }^{7}$ Die Schaffung von Einheitsrecht auf diesem, also legislativem, Weg ist an sich zwar begrüßenswert, zu bedenken gilt jedoch eines: Genauso wie nationale Rechtsvorschriften können auch einheitsrechtliche Vorschriften mehrdeutige oder unbestimmte Gesetzesbegriffe enthalten und damit auslegungsbedürftig sein. ${ }^{8}$ Zur Auslegung dieser Begriffe sind zumeist die nationalen Gerichte der Vertragsstaaten berufen; gemeinsame übergeordnete Gerichte existieren kaum. ${ }^{9}$ Mit der Auslegung des Einheitsrechtes durch die nationalen Gerichte geht nun allerdings eine Gefahr einher, nämlich jene der unterschiedlichen Auslegung. Legen nationale Gerichte Einheitsrecht unterschiedlich aus, kommt es zu dessen „Re-Nationalisierung ". ${ }^{10}$ Es entstehen wiederum nationale Unterschiede, die durch die Vereinheitlichung aber eben gerade beseitigt werden hätten sollen. Die vollkommene Vereinheitlichung einer Rechtsmaterie kann daher nur dann erreicht werden, wenn

\footnotetext{
${ }^{7}$ So bezeichnet etwa Von Bar den Staatsvertrag - zwar im Zusammenhang mit dem Haftungsrecht, aber immerhin - als „,klassisches' Instrument der Rechtsvereinheitlichung“, ZfRV 1994, 221 (222); dem dürfte wohl zumindest auf globaler Ebene nach wie vor so sein, für regionale Rechtsvereinheitlichung mag vielleicht mittlerweile etwas anderes zutreffen. Für eine Übersicht der Rechtsquellen der internationalen Güterbeförderung siehe Zehetbauer/Motter, Nationales und Internationales Transportrecht (2017) 19-21.

${ }^{8}$ Inwiefern Bedarf nach Auslegung besteht, hängt klarerweise auch vom Abstraktionsgrad einer Vorschrift und damit vom gewählten Gesetzesstil ab. Ganz allgemein zum Unterschied zwischen generell-abstraktem und kasuistischem Gesetzesstil siehe Leipold, BGB I: Einführung und allgemeiner Teil ${ }^{10}$ (2019) 22.

${ }^{9}$ Dazu und grundsätzlich zur Auslegungskompetenz siehe Linhart, Einheitsrecht und einheitliche Auslegung (2005) 31-32.

${ }^{10}$ Kramer, Uniforme Interpretation von Einheitsprivatrecht - mit besonderer Berücksichtigung von Art 7 UNKR, JBl 1996, 137 (139); Linhart 2.
} 
Einheitsrecht von den nationalen Gerichten auch einheitlich angewendet und ausgelegt wird; die bloße Ausarbeitung eines Normtextes und dessen Übernahme ins nationale Recht reichen demgegenüber nicht. Vor diesem Hintergrund drängen sich nun allerdings weitere Fragen auf wie etwa jene nach den Methoden, mithilfe denen Einheitsrecht interpretiert werden muss. Rein logisch betrachtet setzt eine einheitliche Auslegung eines Gesetzestextes nämlich den Einsatz von einheitlichen Methoden voraus. Es stellt sich mit anderen Worten also die Frage, wie nationale Gerichte bei der Auslegung von Einheitsrecht vorgehen sollen, um den ,internationalen Rechtsanwendungseinklang" ${ }^{11}$ herzustellen. Diese Frage wird in den jeweiligen Vertragswerken häufig nicht oder nur bedingt beantwortet. So enthalten neuere Vertragswerke zwar häufig Bestimmungen über die Auslegung, diese geben aber teilweise eher Ziele statt Methoden vor, in älteren Vertragswerken - wie etwa dem CMR - fehlen Bestimmungen zur Auslegung zum Teil völlig. Etwas eindeutiger in der Methodenfrage scheint demgegenüber die Literatur. So spricht sich das deutschsprachige Schrifttum - soweit ersichtlich - mit gewissen Modifikationen grundsätzlich für die Heranziehung der klassischen kontinentaleuropäischen ${ }^{12}$ Auslegungsmethoden aus, welche um die Rechtsvergleichende Interpretation ergänzt werden sollen. ${ }^{13}$ Im Zuge letzterer wird neben der Vergleichung mit verwandtem Einheitsrecht und den autonomen nationalen Rechten auch die Auseinandersetzung mit der Rechtsprechung und der Literatur anderer Vertragsstaaten zum jeweiligen Vertragswerk gefordert, wobei eine Bindungswirkung ausländischer Entscheidungen richtigerweise verneint wird. ${ }^{14}$ Diese Auslegungsmethode ist an sich zwar sehr zu begrüßen, in der Praxis könnte sich manches aber als Herausforderung erweisen. Vielleicht gestaltet sich der Zugang zu Entscheidungen ausländischer Gerichte schwierig? Vielleicht fehlen dem Auslegenden die notwendigen Sprachkenntnisse? Vielleicht bleibt in der Praxis auch schlichtweg nicht die Zeit, um umfangreiche rechtsvergleichende Recherchen anzustellen? Vielleicht ist vor allem hier die vergleichende Rechtswissenschaft dazu aufgefordert, entsprechende Vorarbeit zu leisten? Fragen wie diese bleiben zwar leider an dieser, hoffentlich aber nicht an anderer Stelle offen. Abschließend ist jedenfalls wiederholt hervorzuheben, dass Rechtsvereinheitlichung eben nicht bei der Ausarbeitung eines harmonisierten Normtextes endet, sondern weit darüber hinausgeht und auch die uniforme Anwendung und Auslegung dieses Normtextes voraussetzt. Mit eben diesem Bereich müssen wir uns künftig (weiterhin) intensiv beschäftigen, zumal nur so die vollkommene Vereinheitlichung des Transportrechtes realisierbar ist.

\footnotetext{
${ }^{11}$ Zum Begriff siehe Kropholler, Internationales Einheitsrecht (1975) $241 \mathrm{f}$.

${ }^{12} \mathrm{MaW}$ : Die im Civil Law-Rechtskreis vorherrschenden Auslegungsmethoden; zur Rechtskreislehre siehe etwa Kischel, Rechtsvergleichung (2015) 217 ff.

${ }^{13}$ Kropholler 260 ff; Linhart 33 ff; Kramer, JB1 1996, 137 (141).

${ }^{14}$ Siehe dazu insbesondere Kropholler 278-283.
} 
Open Access Dieses Kapitel wird unter der Creative Commons Namensnennung 4.0 International Lizenz (http://creativecommons.org/licenses/by/4.0/deed.de) veröffentlicht, welche die Nutzung, Vervielfältigung, Bearbeitung, Verbreitung und Wiedergabe in jeglichem Medium und Format erlaubt, sofern Sie den/die ursprünglichen Autor(en) und die Quelle ordnungsgemäß nennen, einen Link zur Creative Commons Lizenz beifügen und angeben, ob Änderungen vorgenommen wurden.

Die in diesem Kapitel enthaltenen Bilder und sonstiges Drittmaterial unterliegen ebenfalls der genannten Creative Commons Lizenz, sofern sich aus der Abbildungslegende nichts anderes ergibt. Sofern das betreffende Material nicht unter der genannten Creative Commons Lizenz steht und die betreffende Handlung nicht nach gesetzlichen Vorschriften erlaubt ist, ist für die oben aufgeführten Weiterverwendungen des Materials die Einwilligung des jeweiligen Rechteinhabers einzuholen. 